\title{
PROPIEDADES FÍSICO-MECÁNICAS DE ALGUNAS MADERAS NATIVAS CHILENAS CONÍFERAS Y LATIFOLIADAS POR ULTRASONIDO
}

\section{PHYSICAL AND MECHANICAL PROPERTIES OF SOME CHILEAN SOFTWOODS AND HARDWOODS USING ULTRASOUND}

\author{
Erik Baradit ${ }^{1 \star}$, Peter Niemz ${ }^{2}$, Arturo Fernández-Pérez
}

\begin{abstract}
RESUMEN
La utilización de técnicas no destructivas juega un papel importante y complementario en los procesos de caracterización de la madera. En este trabajo el objetivo fue evaluar algunas propiedades físicomecánicas de maderas nativas chilenas por medio de ultrasonido y compararlas con ensayos mecánicos tradicionales de flexión. Las maderas seleccionadas fueron cuatro latifoliadas: tepa (Laureliopsis philipiana), olivillo (Aextoxicon punctatum), laurel (Laurelia sempervirens) y lenga (Nothofagus pumilio), y dos coníferas: alerce (Fitzroya cupressoides) y mañío (Podocarpus nubigena). Para ésto, se utilizaron los equipos de ultrasonido Steinkamp BP5 y Panametric Epoch XT. Los resultados mostraron mayor anisotropía elástica para las latifoliadas que para las coníferas y una relativa simetría en las velocidades de propagación transversal y los módulos de corte a lo largo de las tres principales direcciones del árbol. También los valores medios de los módulos de elasticidad calculados por ultrasonido resultaron entre 25 y $47 \%$ más altos que los medidos por ensayos mecánicos.
\end{abstract}

Palabras Claves: Maderas nativas chilenas, ultrasonido, Módulos de elasticidad, Módulos de corte.

\begin{abstract}
The non-destructive techniques are a very important tool in the process of wood characterization and evaluation. In this work, the objective is the evaluation of physical and mechanical properties of some native Chilean wood species by using the ultrasound techniques and their comparision with the results of typical mechanical tests. The selected woods were four hardwoods: tepa (Laureliopsis philipiana), olivillo (Aextoxicon punctatum), laurel (Laurelia sempervirens) and lenga (Nothofagus pumilio) and two softwoods: alerce (Fitzroya cupressoides) and mañío (Podocarpus nubigena). The equipment used were two types of ultrasound generators: Steinkamp BP5 and Panametric Epoch XT. The results shown a higher anisotropy for hardwoods than for softwoods, and a relatively symmetry for transversal wave propagation velocities and for shear modulus of elasticity, along the principal directions of the tree. At the same time the mean values of Young modulus of elasticity (MOE) obtained by using the ultrasound equipments were a $25-47 \%$ higher than the same modulus measured by mechanical tests.
\end{abstract}

Keywords: Chilean wood species, ultrasound, Modulus of elasticity, Shear moduli.

\footnotetext{
Departamento de Física, Facultad de Ciencias, Universidad del Bío-Bío, Collao 1202, Concepción, Chile.

${ }^{2}$ Eidgenossische Technische Hochschule, Institute for Building Materials, Wood Physics Group, Zurich, Switzerland.

-Autor para correspondencia: ebaradit@ubiobio.cl

Recibido: 11.05.2012 Aceptado: 10.11.2012
} 


\section{INTRODUCCIÓN}

La información que se tiene sobre los valores de todas las constantes elásticas de las maderas nativas y aclimatas en Chile, bajo diferentes condiciones, es muy escasa. Sin embargo, el conocimiento de estas propiedades es de gran importancia cuando se deben tomar decisiones relacionadas con la utilización de los recursos naturales, y por lo tanto es de alto valor en los campos de la ingeniería, construcción, arquitectura y otros. Sólo en algunas excepciones se cuenta con esta información para algunas especies de maderas seleccionadas (DIN 68364 1979), donde por medio de diferentes ensayos mecánicos se determinan las propiedades de dichas especies. Algunos estudios para ciertas coníferas del tipo Picea han sido realizados por investigadores internacionales Neuhaus (1981), Pozgaj et al. (1997), Keunecke et al. (2007, 2008). Respecto a las maderas nacionales, se cuenta con trabajos tales como: Niemz (1995, 1996), Niemz y Aguilera (1995), Karsulovic et al. (2000), Fuentealba y Baradit (2000), Baradit et al. (2006), en donde se han utilizado algunas técnicas no destructivas con diferentes objetivos. Algunos valores de las constantes para maderas chilenas a lo largo de sus tres direcciones principales fueron evaluadas por Sonderegger et al. (2010).

En general, la cantidad de información en forma de valores de constantes elásticas, accesible para un gran número de maderas, es bastante limitada. Sin embargo, algunos parámetros para las tres direcciones principales del árbol han sido obtenidos en antiguos trabajos: Voigt (1928), Hörig (1933), Carrington (1923) y Krabbe (1960), en particular, para algunas especies tropicales. En la mayoría de los casos, las coníferas muestran que el módulo de corte radial-tangencial $\left(G_{R T}\right)$ es aproximadamente un $10 \%$ de los valores del módulo de corte longitudinal-tangencial $\left(G_{L T}\right)$, mientras que en el caso de las latifoliadas, debido a su alta densidad, es de un $40 \%$ del $G_{L T}$ Sin embargo, es difícil concebir la cuantificación de las propiedades elásticas de las maderas considerando sólo estos valores. Bodig y Jayne (1993) enfatizan la gran variabilidad entre las diferentes especies y la influencia de diferentes factores en las características de la madera, así como el contenido de humedad, la temperatura y otros parámetros.

La información relacionada con la caracterización de las propiedades físico-mecánicas de la madera se obtiene generalmente por medio de ensayos mecánicos tradicionales usando diferentes dispositivos, donde la máquina de ensayos tipo Instron juega un papel central. Sin embargo, estos ensayos, en la mayoría de los casos, son lentos y vinculados al uso de una gran cantidad de materia prima. En este sentido, en los últimos años se han utilizado diferentes técnicas no destructivas para la evaluación y caracterización de la madera y sus derivados.

Una técnica importante, rápida, no destructiva y que puede utilizar pequeñas probetas de madera para su caracterización es el ultrasonido (Bucur 2006). Esta técnica ha sido utilizada con diferentes objetivos, en particular, para la determinación de constantes elásticas de la madera (Sonderegger et al. 2010, Baradit et al. 2012, Kranitz et al. 2012) y también la evaluación de materiales compuestos o morteros (Rosell y Cantalapiedra 2011, Rodriguez y Pazini 2003, Lawson et al. 2011).

El objetivo de este trabajo es obtener información de las seis constantes elásticas de cuatro latifoliadas y dos coníferas nacionales, en particular, los módulos de elasticidad de Young y los módulos de corte, a lo largo de los tres ejes principales de la madera utilizando pulsos ultrasónicos. Para el caso de los módulos de elasticidad de Young, los resultados obtenidos se comparan con los obtenidos por medio de ensayos mecánicos en flexión. 


\section{MATERIAL Y MÉTODOS}

El uso de ondas de ultrasonido para la determinación de las características elásticas de la madera tiene como base a la teoría del estado sólido para cristales. De acuerdo a ésta, la madera puede ser asemejada a un cuerpo ortotrópico con tres direcciones principales de simetría (radial, longitudinal y tangencial). Así, las constantes elásticas a lo largo de los ejes pueden ser encontradas de acuerdo a la ecuación de Christoffel:

$$
\begin{aligned}
& E_{i i}=V_{i i}^{2} \cdot \rho \\
& G_{i j}=V_{i j}^{2} \cdot \rho
\end{aligned}
$$

Donde $E$ es el módulo de elasticidad de Young [Pa]; $G$ es el módulo de Corte [Pa]; $V$ es la velocidad de propagación de la onda $[\mathrm{m} / \mathrm{s}] ; \rho$ es la densidad a $20^{\circ} \mathrm{C}$ y $65 \% \mathrm{HR}\left[\mathrm{kg} / \mathrm{m}^{3}\right]$; i es la dirección de propagación de la onda y $j$ es la dirección de oscilación del medio material, las cuales se ajustan de acuerdo a la polarización de la onda ultrasónica utilizada (longitudinal o transversal). Como a lo largo de cada eje pueden propagarse tres ondas: dos de polarización transversal y una de polarización longitudinal, entonces del conjunto de ecuaciones (1) y (2) se obtienen seis constantes: tres módulos de elasticidad y tres módulos de corte.

Las maderas chilenas nativas seleccionadas para este estudio fueron cuatro latifoliadas: tepa (Laureliopsis philipiana), olivillo (Aextoxicon punctatum), laurel (Laurelia sempervirens) y lenga (Nothofagus pumilio); y dos coníferas: alerce (Fitzroya cupressoides) y mañio (Podocarpus nubigena). Estas maderas fueron climatizadas a $12 \%$ de contenido de humedad $\left(\mathrm{A} 20^{\circ} \mathrm{C} / 65 \%\right.$ de humedad relativa de aire) y posteriormente se obtuvieron de ellas probetas libres de defectos, a distancia media entre la médula y la corteza. Las dimensiones fueron de $400 \mathrm{~mm}$ de largo y sección cuadrada de $20 \mathrm{~mm}$ (radial) x $20 \mathrm{~mm}$ (tangencial). Ellas fueron sujetas a experimentación por medio de un equipo de ultrasonido de baja frecuencia $(52 \mathrm{kHz})$ Steinkamp BP5. La cantidad de probetas varió entre 20 y 30, dependiendo de la especie, debido a que fueron seleccionadas las que presentaban solo una clara dirección radial de corte. Durante todo el proceso de medición, se mantuvo constante la presión de los transductores con las probetas, a través de un sistema de acoplamiento adecuado. Usando el tiempo de vuelo y la longitud de las probetas, se obtuvieron los módulos de elasticidad de Young a frecuencias de $52 \mathrm{kHz}$. Para ésto fueron calculadas las densidades aparentes de cada una de las muestras. Estas probetas también fueron experimentadas en flexión para obtener el módulo de elasticidad y el módulo de ruptura por ensayo mecánico según la norma DIN 52186 (1978).

También fueron obtenidas probetas cúbicas de todas las especies, de $20 \mathrm{~mm}$ de arista en las 3 direcciones principales (longitudinal, radial y transversal). Éstas fueron experimentadas con un equipo generador de ultrasonido Panametric Epoch XT con transductores de ondas longitudinales de 2,25 $\mathrm{MHz}$ (Olympus A133S) y de ondas transversales de $1 \mathrm{MHz}$ (Staveley S-0104). En estas mediciones, se utilizó el gel de acoplamiento específico Ultrasonic Couplant II, entre las probetas y los transductores. 


\section{RESULTADOS Y DISCUSIÓN}

Los resultados de este trabajo se resumen en las tablas 1-4. En la tabla 1, se muestran los valores obtenidos para los módulos de elasticidad en mediciones con ultrasonido $\left(M O E_{U S}\right)$, usando una frecuencia de $52 \mathrm{kHz}$ con el equipo Steinkamp BP5 y sólo a lo largo de la dirección longitudinal del árbol. Además, se midieron los módulos de elasticidad y de ruptura estáticos $\left(M O E_{E S} \mathrm{y} M O R\right)$, mediante ensayos mecánicos. Se observa que los valores de los módulos de elasticidad obtenidos por medio de ultrasonido (BP5, 52 $\mathrm{kHz}$ ) son para el caso de las coníferas, entre un 25 a un $28 \%$ más altos que los obtenidos por ensayos estáticos. Para el caso de las latifoliadas, la diferencia es entre un 30\% a un $47 \%$. De esta manera, tanto para las coníferas como para las latifoliadas se cumple que $M O E_{U S}>M O E_{E S}$.

Por otro lado, se observa también una clara diferencia entre los valores de las dos coníferas mañío y alerce. Éste último muestra valores más bajos en las velocidades de propagación de polarización longitudinal y en los valores de los módulos de elasticidad de Young (esta diferencia es menor para los ondas de corte). Nótese que la densidad del alerce es menor que la del mañío. En la figura 1 se muestran imágenes por microscopía electrónica de cada una las especies, en la cuál se aprecia una mayor proporción de pared celular en el mañío, lo que condiciona una mejor transmisión de las ondas en esta especie en relación con el alerce. Esta propiedad a su vez se refleja en un mayor módulo de elasticidad para el mañio. Cabe notar que, diferencias similares fueron encontradas por Niemz y Aguilera (1995) y Díaz Vaz et al. (1989).

Tabla 1. Densidad, Velocidad de Propagación y Módulos de elasticidad y de ruptura a condiciones climáticas estándar $\left(20^{\circ} \mathrm{C} / 65 \% \mathrm{HR}\right)$ a lo largo de la dirección longitudinal del árbol.

\begin{tabular}{|c|c|c|c|c|c|c|}
\hline \multicolumn{2}{|l|}{ Especie } & \multirow{2}{*}{$\begin{array}{c}\begin{array}{c}\text { Densidad } \\
\left(\mathbf{k g} / \mathbf{m}^{3}\right)\end{array} \\
549\end{array}$} & \multirow{2}{*}{$\begin{array}{c}\begin{array}{c}\text { Velocidad de } \\
\text { propagación } \\
(\mathrm{m} / \mathrm{s})\end{array} \\
4641\end{array}$} & \multirow{2}{*}{$\begin{array}{c}\text { MOE }_{\text {Us }} \\
\text { Ultrasonido } \\
\text { (MPa) }\end{array}$} & \multirow{2}{*}{$\begin{array}{c}\text { MOE }_{\mathrm{ES}} \\
\text { Estático } \\
\text { (MPa) }\end{array}$} & \multirow{2}{*}{$\begin{array}{r}\text { MOR } \\
\text { (MPa) } \\
72 \\
\end{array}$} \\
\hline & $x$ & & & & & \\
\hline \multirow{3}{*}{$\begin{array}{c}\text { Tepa } \\
\text { (Laureliopsis } \\
\text { philipiana) }\end{array}$} & $s$ & 19 & 244 & 1209 & 1136 & 9 \\
\hline & $c v$ & 3.5 & 5.2 & 10.4 & 14.3 & 12.3 \\
\hline & $x$ & 509 & 4630 & 11026 & 8043 & 64 \\
\hline \multirow{3}{*}{$\begin{array}{c}\text { Olivillo } \\
\text { (Aextoxicon } \\
\text { punctatum) }\end{array}$} & $s$ & 57 & 187 & 1919 & 1358 & 7 \\
\hline & $c v$ & 11.1 & 4.0 & 17.4 & 16.9 & 11.1 \\
\hline & $x$ & 553 & 4850 & 12966 & 9979 & 88 \\
\hline \multirow{3}{*}{$\begin{array}{c}\text { Laurel } \\
\text { (Laurelia } \\
\text { sempervirens) }\end{array}$} & $s$ & 18 & 140 & 709 & 610 & 9 \\
\hline & $c v$ & 3.3 & 2.8 & 5.5 & 6.1 & 9.7 \\
\hline & $x$ & 545 & 4508 & 10808 & 8053 & 72 \\
\hline \multirow{3}{*}{$\begin{array}{c}\text { Lenga } \\
\text { (Nothofagus } \\
\text { pumilio) }\end{array}$} & $s$ & 50 & 363 & 903 & 789 & 5 \\
\hline & $c v$ & 9.2 & 8.1 & 8.4 & 9.8 & 6.4 \\
\hline & $x$ & 407 & 4410 & 7910 & 6169 & 61 \\
\hline \multirow{3}{*}{$\begin{array}{c}\text { Alerce } \\
\text { (Fitzroya } \\
\text { cupressoides) }\end{array}$} & $s$ & 10 & 60 & 379 & 287 & 6 \\
\hline & $c v$ & 2.5 & 1.4 & 4.8 & 4.6 & 10.4 \\
\hline & $x$ & 569 & 4780 & 13043 & 10460 & 102 \\
\hline \multirow{2}{*}{$\begin{array}{c}\text { Mañío } \\
\text { (Podocarpus } \\
\text { nubigena) }\end{array}$} & $s$ & 43 & 260 & 1823 & 1336 & 12 \\
\hline & $c v$ & 7.6 & 5.5 & 14.0 & 12.8 & 11.8 \\
\hline
\end{tabular}

x - valor medio, s - desviación estándar, cv - coeficiente de variación. 
Por otro lado, en el caso de las latifoliadas, no se observan grandes diferencias tanto en los valores de las velocidades de propagación como en los $M O E$, tanto en los obtenidos por ensayos mecánicos y por ultrasonido. La mayor diferencia ocurre entre estas especies y el laurel, dado que este último tiene menor proporción de pared celular (Figura 1), mostrando así un mayor valor en los módulos de elasticidad y las velocidades de propagación.
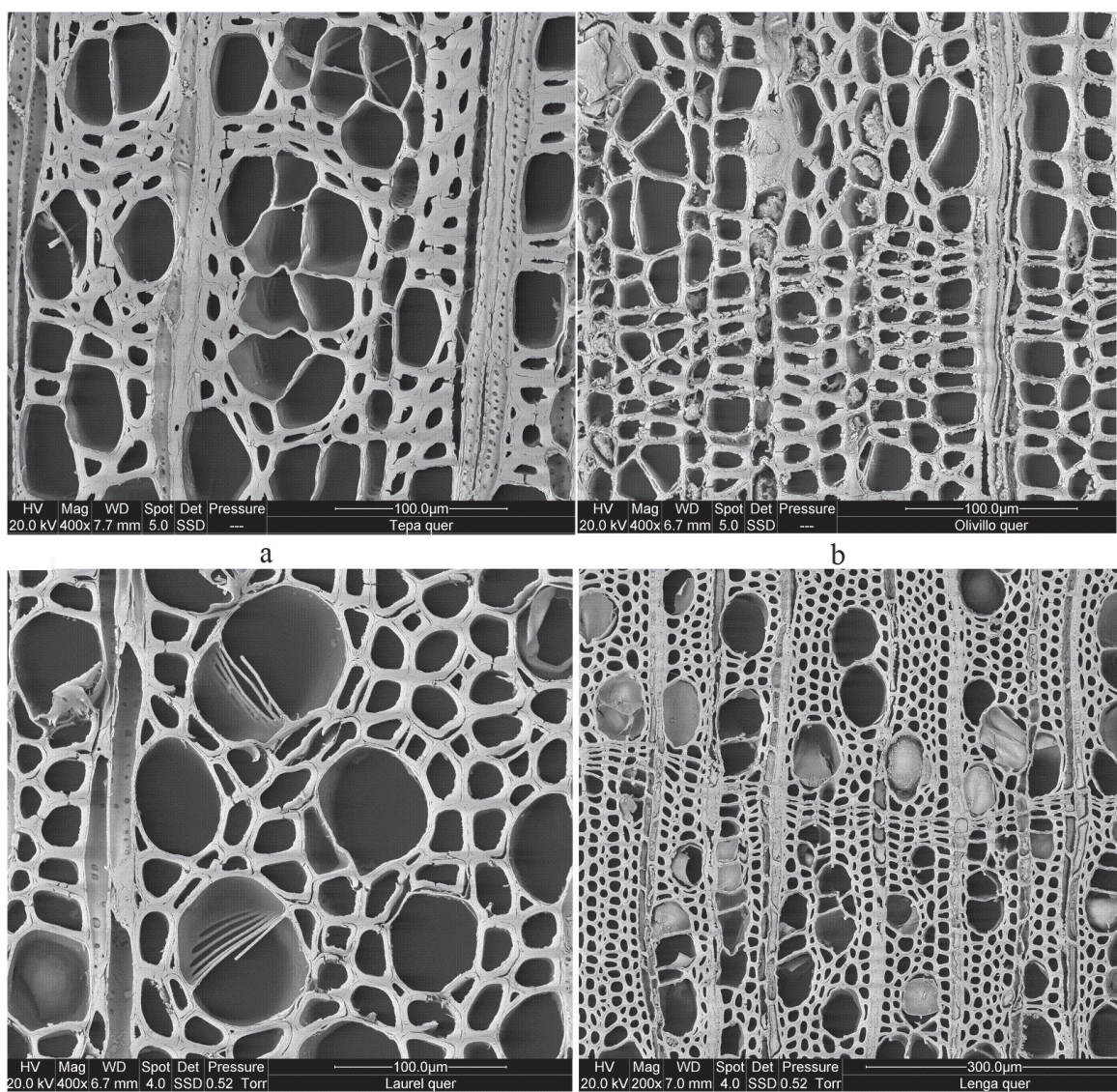

b

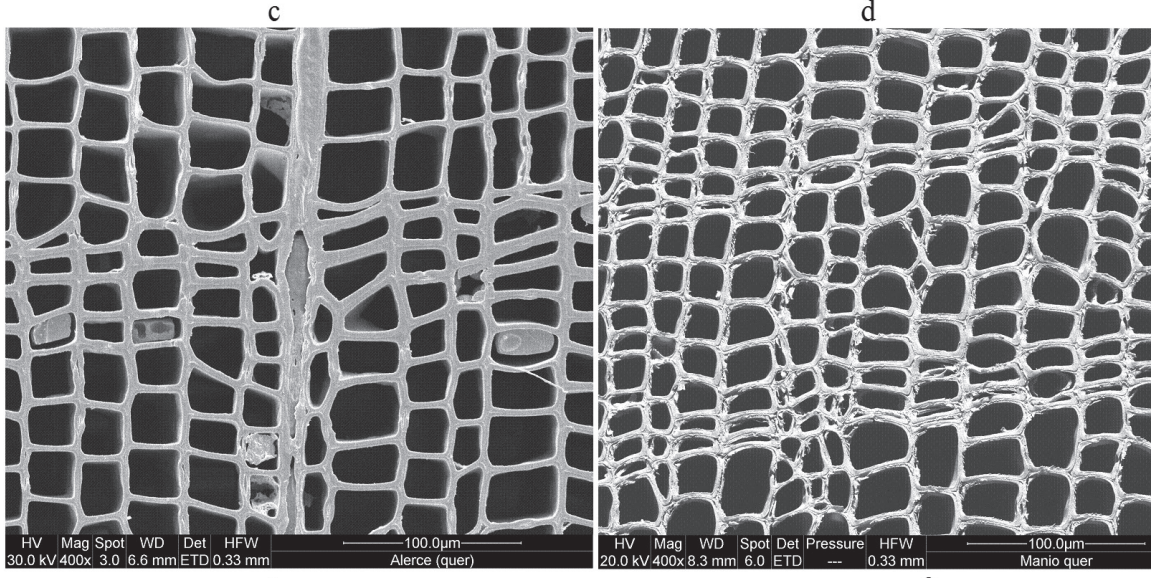

e

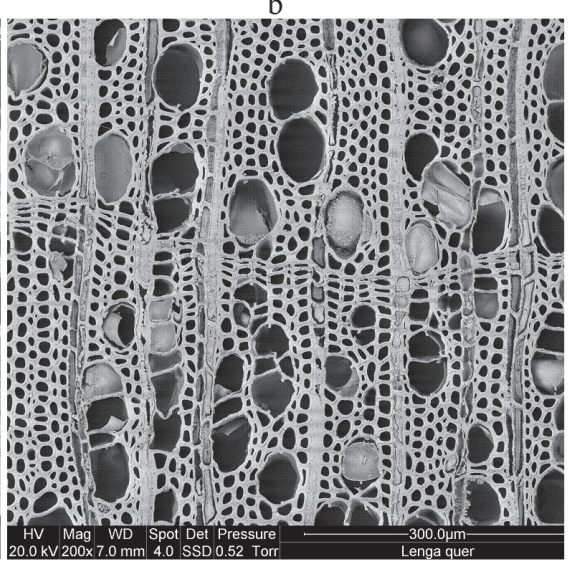

d

Figura 1. Imágenes de microscopía electrónica (corte transversal) de las maderas latifoliadas

a) Tepa (Laureliopsis philipiana); b) Olivillo (Aextoxicon punctatum);

c) Laurel (Laurelia sempervirens); d) Lenga (Nothofagus pumilio); y de las coníferas

e) Alerce (Fitzroya cupressoides); f) Mañío(Podocarpus nubigena). 
En la tabla 2 se observa que los valores de velocidad de propagación a lo largo de la dirección longitudinal de las probetas cúbicas medidos con el equipo Epoch XT, son similares a aquellos obtenidos en las barras y medidos con el Steinkamp BP5 (Tabla 1). El alerce mostró los valores más bajos de propagación en las direcciones longitudinal $\left(\mathrm{V}_{\mathrm{LL}}\right)$ y radial $\left(\mathrm{V}_{\mathrm{RR}}\right)$, mientras que en la dirección tangencial $\left(\mathrm{V}_{\mathrm{TT}}\right)$, estos valores fueron más variados entre las especies. Tanto usando el BP5 como el Epoch XT los resultados mostraron para el alerce valores más bajos para el Módulo de elasticidad de Young en la dirección longitudinal $\left(\mathrm{E}_{\mathrm{LL}}\right)$ y los $M O E$ estáticos (Tablas 1 y 2).

Tabla 2. Densidad, velocidad de propagación y Módulos de elasticidad a condiciones climáticas estándar $\left(20^{\circ} \mathrm{C} / 65 \% \mathrm{HR}\right)$ a lo largo de las tres direcciones principales.

\begin{tabular}{|c|c|c|c|c|c|c|c|c|}
\hline \multicolumn{2}{|l|}{ Especie } & \multirow{2}{*}{$\begin{array}{c}\begin{array}{c}\text { Densidad } \\
\left(\mathbf{k g} / \mathbf{m}^{3}\right)\end{array} \\
560\end{array}$} & \multirow{2}{*}{$\begin{array}{c}\begin{array}{c}\mathbf{V}_{\mathbf{L L}} \\
(\mathbf{m} / \mathbf{s})\end{array} \\
4684\end{array}$} & \multirow{2}{*}{$\begin{array}{c}\begin{array}{c}\mathbf{V}_{\mathrm{RR}} \\
(\mathbf{m} / \mathbf{s})\end{array} \\
2052\end{array}$} & \multirow{2}{*}{$\begin{array}{l}\begin{array}{l}\mathbf{V}_{\mathrm{TT}} \\
(\mathrm{m} / \mathrm{s})\end{array} \\
1163\end{array}$} & \multirow{2}{*}{$\begin{array}{r}\begin{array}{c}\mathbf{E}_{\mathrm{LL}} \\
\text { (MPa) }\end{array} \\
12371\end{array}$} & \multirow{2}{*}{$\begin{array}{c}\begin{array}{c}\mathbf{E}_{\mathbf{R R}} \\
(\mathbf{M P a})\end{array} \\
2360\end{array}$} & \multirow{2}{*}{$\begin{array}{r}\begin{array}{c}\mathbf{E}_{\mathrm{TT}} \\
\text { (MPa) }\end{array} \\
758\end{array}$} \\
\hline & $x$ & & & & & & & \\
\hline \multirow{3}{*}{$\begin{array}{c}\text { Tepa } \\
\text { (Laureliopsis } \\
\text { philipiana) }\end{array}$} & $s$ & 16 & 281 & 64 & 36 & 1783 & 152 & 36 \\
\hline & $c v$ & 2,85 & 6 & 3,12 & 3,09 & 14,41 & 6,44 & 4,75 \\
\hline & $x$ & 546 & 4857 & 1942 & 1119 & 13046 & 2043 & 685 \\
\hline \multirow{3}{*}{$\begin{array}{l}\text { Olivillo } \\
\text { (Aextoxicon } \\
\text { punctatum) }\end{array}$} & $s$ & 7 & 291 & 123 & 63 & 2867 & 148 & 106 \\
\hline & $c v$ & 12,82 & 5,99 & 6,33 & 5,6 & 21,9 & 7,2 & 15,47 \\
\hline & $x$ & 472 & 4900 & 2050 & 1211 & 11391 & 1982 & 692 \\
\hline \multirow{3}{*}{$\begin{array}{c}\text { Laurel } \\
\text { (Laurelia } \\
\text { sempervirens) }\end{array}$} & $s$ & 29 & 217 & 63 & 33 & 1436 & 137 & 48 \\
\hline & $c v$ & 6,14 & 4,43 & 3,07 & 2,72 & 12,6 & 6,91 & 6,5 \\
\hline & $x$ & 512 & 4918 & 2078 & 1190 & 12380 & 2215 & 727 \\
\hline \multirow{2}{*}{$\begin{array}{c}\text { Lenga } \\
\text { (Nothofagus } \\
\text { pumilio) }\end{array}$} & $s$ & 27 & 101 & 56 & 47 & 758 & 205 & 70 \\
\hline & $c v$ & 5,27 & 2,05 & 2,69 & 3,95 & 6,12 & 9,25 & 10,72 \\
\hline \multirow{4}{*}{$\begin{array}{c}\text { Alerce } \\
\text { (Fitzroya } \\
\text { cupressoides) }\end{array}$} & $x$ & 412 & 4503 & 1865 & 1440 & 8732 & 1466 & 858 \\
\hline & $s$ & 19 & 108 & 267 & 61 & 1027 & 450 & 90 \\
\hline & $c v$ & 4,6 & 4,1 & 14 & 4,2 & 11,8 & 30,7 & 10,4 \\
\hline & $x$ & 561 & 5000 & 2153 & 1610 & 14027 & 2599 & 1465 \\
\hline \multirow{2}{*}{$\begin{array}{c}\text { Mañío } \\
\text { (Podocarpus } \\
\text { nubigena) }\end{array}$} & $s$ & 21 & 54 & 51 & 145 & 975 & 149 & 279 \\
\hline & $c v$ & 3,74 & 3,08 & 2,37 & 9,01 & 6,95 & 5,73 & 19 \\
\hline
\end{tabular}

$\mathrm{x}$ - valor medio, s - desviación estándar, cv - coeficiente de variación.

V: Velocidad de Propagación; E: Modulo de Elasticidad; L: dirección longitudinal; R: dirección radial;

T: dirección tangencial 
Un resultado importante es mostrado en la tabla 3, donde se especifican las velocidades obtenidas por medio de mediciones con el equipo Epoch XT de ondas transversales, se aprecia una relativa simetría en las velocidades transversales $\left(V_{i j}=V_{j i}\right)$. Debido a ésto $\left(G_{i j}=G_{j i}\right)$ se calcularon los valores promedio de $G_{L T}$ y $G_{T L} ; G_{L R}$ y $G_{R L} ; G_{R T}$ y $G_{T R}$. Sin embargo, los resultados numéricos para $G_{i j}$ mostraron que la simetría de los valores es aproximada y no exacta (Bucur 2006). Los valores de $G_{R T}$ obtenidos fueron un poco más altos de lo esperado, sin embargo, las relaciones entre los módulos de corte $\left(G_{L R}: G_{L T}\right.$ : $\left.G_{R T}\right)$ para el alerce fue de 10:9:1, mientras que para las maderas latifoliadas $G_{L T}$ represento ser entre un $40-45 \%$ menor que $G_{L R}$, lo que es similar a lo encontrado por Bodig y Jayne (1993).

Tabla 3. Densidad, velocidad de propagación y Módulos de corte Gij, en condiciones climáticas estándar $\left(20^{\circ} \mathrm{C} / 65 \% \mathrm{HR}\right)$, a lo largo de las tres direcciones principales.

\begin{tabular}{|c|c|c|c|c|c|c|c|c|c|c|c|}
\hline \multicolumn{2}{|l|}{ Especie } & \multirow{2}{*}{$\begin{array}{c}\begin{array}{c}\text { Densidad } \\
\left(\mathrm{kg} / \mathrm{m}^{3}\right)\end{array} \\
560\end{array}$} & \multirow{2}{*}{$\begin{array}{c}\begin{array}{c}V_{L R} \\
(\mathrm{~m} / \mathbf{s})\end{array} \\
1440\end{array}$} & \multirow{2}{*}{$\begin{array}{c}\begin{array}{c}V_{R L} \\
(\mathrm{~m} / \mathbf{s})\end{array} \\
1324\end{array}$} & \multirow{2}{*}{$\begin{array}{r}V_{L T} \\
(\mathrm{~m} / \mathrm{s})\end{array}$} & \multirow{2}{*}{$\begin{array}{c}\begin{array}{c}V_{T L} \\
(\mathrm{~m} / \mathrm{s})\end{array} \\
1083\end{array}$} & \multirow{2}{*}{$\begin{array}{c}V_{R T} \\
(\mathrm{~m} / \mathrm{s})\end{array}$} & \multirow{2}{*}{$\begin{array}{c}\begin{array}{c}V_{T R} \\
(\mathrm{~m} / \mathrm{s})\end{array} \\
750\end{array}$} & \multirow{2}{*}{$\begin{array}{c}\begin{array}{c}G_{L R} \\
(\mathbf{M P a})\end{array} \\
1074\end{array}$} & \multirow{2}{*}{$\begin{array}{c}\begin{array}{c}G_{L T} \\
(\mathbf{M P a})\end{array} \\
725\end{array}$} & \multirow{2}{*}{$\begin{array}{c}\begin{array}{c}\boldsymbol{G}_{\boldsymbol{R} T} \\
(\mathbf{M P a})\end{array} \\
312\end{array}$} \\
\hline & $x$ & & & & & & & & & & \\
\hline \multirow{3}{*}{$\begin{array}{c}\text { Tepa } \\
\text { (Laureliopsis } \\
\text { philipiana) }\end{array}$} & $s$ & 16 & 30 & 80 & 24 & 61 & 21 & 35 & 97 & 54 & 20 \\
\hline & $c v$ & 2,8 & 2,1 & 6 & 2 & 5,6 & 2,8 & 4,7 & 9 & 7,4 & 6,4 \\
\hline & $x$ & 546 & 1495 & 1506 & 1188 & 1082 & 613 & 616 & 1237 & 705 & 209 \\
\hline \multirow{3}{*}{$\begin{array}{c}\text { Olivillo } \\
\text { (Aextoxicon } \\
\text { punctatum) }\end{array}$} & $S$ & 7 & 47 & 74 & 26 & 33 & 23 & 45 & 219 & 90 & 45 \\
\hline & $c v$ & 12,8 & 3,1 & 4,9 & 2,2 & 3 & 3,7 & 7,3 & 17,7 & 12,8 & 21,5 \\
\hline & $x$ & 472 & 1497 & 1531 & 1239 & 1127 & 655 & 656 & 1084 & 663 & 203 \\
\hline \multirow{3}{*}{$\begin{array}{c}\text { Laurel } \\
\text { (Laurelia } \\
\text { sempervirens) }\end{array}$} & $s$ & 29 & 50 & 50 & 30 & 34 & 8 & 17 & 107 & 65 & 13 \\
\hline & $c v$ & 6,1 & 3,3 & 3,3 & 2,4 & 3 & 1,2 & 2,6 & 9,9 & 9,8 & 6,4 \\
\hline & $x$ & 512 & 1573 & 1583 & 1207 & 1126 & 625 & 595 & 1277 & 699 & 192 \\
\hline \multirow{3}{*}{$\begin{array}{c}\text { Lenga } \\
\text { (Nothofagus } \\
\text { pumilio) }\end{array}$} & $s$ & 27 & 48 & 51 & 40 & 36 & 25 & 55 & 122 & 67 & 29 \\
\hline & $c v$ & 5,3 & 3 & 3,2 & 3,3 & 3,2 & 4 & 9,2 & 9,5 & 9,6 & 15 \\
\hline & $x$ & 412 & 1674 & 1554 & 1533 & 1508 & 624 & 592 & 1547 & 1398 & 158 \\
\hline \multirow{3}{*}{$\begin{array}{c}\text { Alerce } \\
\text { (Fitzroya } \\
\text { cupressoides) }\end{array}$} & $s$ & 19 & 45 & 120 & 44 & 54 & 125 & 35 & 149 & 71 & 37 \\
\hline & $c v$ & 4,6 & 2,7 & 7,7 & 2,9 & 3,6 & 20 & 6,0 & 9,6 & 5,1 & 23 \\
\hline & $x$ & 561 & 1632 & 1678 & 1550 & 1601 & 667 & 651 & 1081 & 954 & 232 \\
\hline \multirow{2}{*}{$\begin{array}{c}\text { Mañío } \\
\text { (Podocarpus } \\
\text { nubigena) }\end{array}$} & $s$ & 21 & 66 & 162 & 98 & 140 & 78 & 67 & 246 & 214 & 65 \\
\hline & $c v$ & 3,74 & 4 & 9,6 & 6,3 & 8,7 & 11,7 & 10,3 & 22,7 & 22,4 & 28 \\
\hline
\end{tabular}

x - valor medio, s - desviación estándar, cv - coeficiente de variación.

V: Velocidad de Propagación; G: Modulo de Corte; L: dirección longitudinal; R: dirección radial; $\mathrm{T}$ : dirección tangencial 
Los valores para las relaciones entre las velocidades de propagación y entre los módulos de elasticidad, para las tres principales direcciones del árbol, se presentan en la tabla 4. Se observa una mayor diferencia en los valores de los módulos y en las velocidades de propagación (con polarización longitudinal) para las latifoliadas que para las coníferas. Ésto es contrario a lo presentado por Bucur (2006) quién encontró mayores diferencias en los valores para las coníferas. Por ejemplo, para el alerce se observan las menores diferencias en los valores para las tres direcciones principales. Ésta diferencia es menor a lo publicado por Bodig y Jayne (1993) y también a Von Halasz y Scheer (1986). Ésto podría deberse a la poca diferencia entre la densidad de madera inicial y tardía. Éstas pequeñas variaciones para el alerce también fueron encontradas por Niemz y Aguilera (1995). Resultados similares e incluso valores más bajos fueron encontrados por Bucur (2006) para diferentes coníferas y por Keunecke et al. (2007) para la Picea de Noruega (Picea abies).

Tabla 4. Relaciones entre las velocidades y los módulos de elasticidad longitudinales.

\begin{tabular}{|c|c|c|}
\hline Especie & $\begin{array}{l}\text { Razón entre las } \\
\text { velocidades } \\
\mathrm{V}_{\mathrm{TT}}: \mathrm{V}_{\mathrm{RR}}: \mathrm{V}_{\mathrm{LL}}\end{array}$ & $\begin{array}{l}\text { Razón entre los módulos de Young } \\
\qquad \mathrm{E}_{\mathrm{TT}}: \mathrm{E}_{\mathrm{RR}}: \mathrm{E}_{\mathrm{LL}}\end{array}$ \\
\hline $\begin{array}{c}\text { Tepa } \\
\text { (Laureliopsis philipiana) }\end{array}$ & $1: 2.3: 4.0$ & $1: 3.1: 16.3$ \\
\hline $\begin{array}{c}\text { Olivillo } \\
\text { (Aextoxicon punctatum) }\end{array}$ & $1: 2.5: 4.3$ & $1: 3.0: 19.0$ \\
\hline $\begin{array}{c}\text { Laurel } \\
\text { (Laurelia sempervirens) }\end{array}$ & $1: 2.4: 4.0$ & $1: 2.9: 16.5$ \\
\hline $\begin{array}{c}\text { Lenga } \\
\text { (Nothofagus pumilio) }\end{array}$ & $1: 2.3: 4.1$ & $1: 3.0: 17.0$ \\
\hline $\begin{array}{c}\text { Alerce } \\
\text { (Fitzroya cupressoides) }\end{array}$ & $1: 2.4: 3.1$ & $1: 1.7: 10.2$ \\
\hline $\begin{array}{c}\text { Mañío } \\
\text { (Podocarpus nubigena) }\end{array}$ & $1: 2.3: 3.1$ & $1: 1.7: 9.6$ \\
\hline
\end{tabular}

V: Velocidad de Propagación; E: Modulo de Elasticidad; L: dirección longitudinal; $\mathrm{R}$ : dirección radial; $\mathrm{T}$ : dirección tangencial

Los resultados muestran que los módulos de elasticidad medios obtenidos en base de mediciones con ondas de propagación de ultrasonido son más altos (entre un 25 - $47 \%$ ) que los recogidos por medio de ensayos mecánicos en flexión, lo que es una relación común para estos dos métodos. Por otro lado, las relaciones obtenidas entre los módulos de elasticidad longitudinales y tangenciales para las latifoliadas son similares a las relaciones generales encontradas por Bodig y Jayne (1993), mostradas en la tabla 5. Sin embargo, se observa una diferencia en las relaciones con el módulo radial. Por otro lado, para las coníferas se observa una similitud en las relaciones entre los módulos de elasticidad radial y tangencial, y no en las relaciones con el módulo longitudinal. Por lo tanto, las relaciones entre los módulos de corte obtenidas en este estudio para cada especie son diferentes a las mostradas por Bodig y Jayne (1993) y sólo en el caso del alerce (Fitzroya cupressoides) se observa una similitud en los resultados. 
Tabla 5. Correlaciones generales para los módulos de elasticidad y de corte.

\begin{tabular}{c}
$\frac{\mathrm{E}_{\mathrm{L}}: \mathrm{E}_{\mathrm{R}}: \mathrm{E}_{\mathrm{T}} \cdot 20: 1.6: 1}{\mathrm{G}_{\mathrm{LR}}: \mathrm{G}_{\mathrm{LT}}: \mathrm{G}_{\mathrm{RT}} \cdot 10: 9.4: 1}$ \\
\hline $\mathrm{E}_{\mathrm{L}}: \mathrm{G}_{\mathrm{LR}}=14: 1$
\end{tabular}

E: Modulo de Elasticidad; G: Modulo de Corte; L: dirección longitudinal; R: dirección radial; T: dirección tangencial

( Bodig y Jayne 1993)

En complementación a ésto, se observa una clara diferencia para las coníferas, en los valores para las velocidades de propagación y los módulos de elasticidad, debido principalmente a la estructura anatómica del alerce. Además, los resultados obtenidos mostraron que las maderas nativas chilenas tienen una mayor razón de anisotropía para las latífoliadas que para las coníferas, diferenciándose en ésto de varias maderas europeas, que muestran lo contrario. Finalmente, se encuentra una diferencia entre las constantes elásticas a lo largo de los ejes principales y una simetría relativa, tanto para las velocidades de propagación de polarización transversal como para módulos de corte.

\section{CONCLUSIÓN}

El uso de una técnica no destructiva como el ultrasonido, permitió una evaluación de los módulos de elasticidad y de corte para seis maderas nativas chilenas, encontrándose en ellas relaciones características para las tres direcciones principales del árbol. Los resultados mostraron para las latifoliadas variaciones entre un $30 \%$ a un $47 \%$ en los valores de los $M O E$ obtenidos por ultrasonido y por ensayos mecánicos, mientras que para las coníferas esta diferencia fue de entre un $25 \%$ a un $28 \%$. Al mismo tiempo, se obtuvo una buena simetría en los valores para las velocidades de ondas transversales para los tres planos, por lo que se calcularon los valores promedio de los módulos de corte $G_{i: .}$. Finalmente, se muestra que las latifoliadas tienen mayor anisotropía a lo largo de los ejes principales del árbol, que las coníferas, como se puede observar de las relaciones que existen entre los módulos de Young para las diferentes especies.

\section{AGRADECIMIENTOS}

Los autores agradecen al Instituto de Materiales de Construcción de la ETH de Zurich por la facilitación del uso de laboratorios, equipos y espacios en el desarrollo de la investigación. Al mismo tiempo agradecen el apoyo y ayuda de Tomas Schneider, ETH en el proceso de medición.

\section{REFERENCIAS}

Baradit, E.; Aedo, R.; Correa, J. 2006. Knot detection in wood using microwaves. Wood Science and Technology 40 (2):118-123.

Baradit, E.; Keunecke, D.; Niemz, P. 2012. Stifness Moduli of various extraneous species determined with ultrasound. Wood Research 57(1):173-178.

Bodig, J.; Jayne, B. A. 1993. Mechanics of wood and wood composites. Krieger Publishing Company, Malabar.

Bucur, V. 2006. Acoustics of Wood. Springer, Berlin. 39-50

Carrington, H. 1923. The elastic constants of spruce. Philosophical Magazine 45 (269):1055-1057.

Diaz Vaz, J. E.; Devlieger, F.; Poblete, H.; Juacida, R. 1989. Maderas Comerciales de Chile. Marisa Cueno Ediciones, Valdivia. 91 p. 
DIN. Deutsches Institut Fur Normung. 1978. Testing of wood; bending test. DIN 52186. BeuthVerlag. Berlín.

DIN. Deutsches Institut Fur Normung. 1979. Characteristic values for wood species; strength, elasticity, resistance. DIN 68364. Beuth-Verlag, Berlín.

Fuentealba, C.; Baradit, E. 2000. Determinación de las constantes elásticas de la madera por ultrasonido: Parte I: Módulos de elasticidad y módulos de rigidez. Maderas. Ciencia y Tecnología 2(2):161-167.

Hörig, H. 1933. Zur Elastizität des Fichtenholzes. 1. Folgerungen aus Messungen von H. Carrington an Spruce. Zeitschrift für technische Physik 12: 369-379.

Keunecke, D.; Sonderegger, W.; Pereteanu, K.; Lüthi, T.; Niemz, P. 2007. Determination of Young's and shear moduli of common yew and Norway spruce by means of ultrasonic waves. Wood Science and Technology 41(4):309-327.

Keunecke, D.; Hering, S.; Niemz, P. 2008. Three-dimensional elastic behaviour of common yew and Norway spruce. Wood Science and Technology 42 (8):633-647

Karsulovic, J. T.; León, L. A.; Gaete, L. 2000. Ultrasonic detection of knots and annual ring orientation in Pinus radiata lumber. Wood and Fiber Science 32(3):278-286.

Krabbe, E. 1960. Messungen von Gleit- und Dehnungszahlen an Holzstäbchen mit rechteckigen Querschnitten. Phd Thesis, Technische Hochschule Hannover, Hannover, Alemania, 106p.

Kranitz, K.; Baradit, E.; Dobrowolska, E.; Plotze, M.; Niemz, P. 2012. Untersuchungen zu Eingenschaften von Mooreiche. Holztechnologie 53(1):11-17.

Lawson, I.; Danso, H.C.; Odoi, H.C.; Adjei, C.A.; Quashie, I.I.; Mumuni, I.I.; Ibrahim, I.S. 2011. Non-Destructive evaluation of concrete using ultrasound pulse velocity. Research Journal of Applied Sciences, Engeneering and Technology 3(6):499-504.

Neuhaus, F. H. 1981. Elastizitätszahlen von Fichtenholz in Abhängigkeit von der Holzfeuchtigkeit. Bochum, Phd Thesis, Institut für Konstruktiven Ingenieurbau Ruhr-Universität Bochum, Alemania 146p.

Niemz, P. 1995. Schallausbreitungsgeschwindigkeit einiger chilenischer Holzarten. Holz als Rohund Werkstoff 53 (2):100.

Niemz, P.; Aguilera, A. 1995. Untersuchungen zur Schallausbreitungsgeschwindigkeit für ausgewählte Holzarten Chiles. Holz als Roh- und Werkstoff 53 (3):187-191.

Niemz, P. 1996. Untersuchungen zum Einfluss der Holzfeuchte aufdie Schallausbreitungsgeschwindigkeit in Roble. Holz als Roh- und Werkstoff 54(1):60.

Pozgaj, A.; Chovanec, D.; Kurjatko, S.; Babiak, M. 1997. Štruktúra a vlastnosti dreva. Príroda, Bratislava. ISBN 8007009604. 485p

Rodríguez, G.S.; Pazini, E. 2003. Módulo de elasticidad estático del hormigón determinado por medio de pruebas de ultrasonido. Materiales de Construcción 53(271-272): 47-55.

Rosell, J.R.; Cantalapiedra, I.R. 2011. Método simple para determinar el módulo de Young dinámico a partir de una exitación por impacto aplicado a morteros de cal y cemento. Materiales de Construcción 61(301):9-48.

Sonderegger, W.; Keunecke, D.; Baradit, E.; Niemz, P. 2010. Selected physical and mechanical properties of Chilean wood species roble, lingue, mañío and alerce. Wood Material Science and Engineering 5(1):53-59.

Voigt, W. 1928. Lehrbuch der Kristallphysik. Johnson Reprint Corp. Teubner, Leipzig.

Von Halasz, R.; Scheer, C. 1986. Holzbau-Taschenbuch. Verlag Ernst \& Sohn. Berlin. 